\title{
Photoemission study of the Al-Sb(111) interface
}

\author{
R. Sporken, P. A. Thiry, E. Petit, J. J. Pireaux, and R. Caudano \\ Laboratoire de Spectroscopie Electronique, Institut de Recherche sur les Interfaces Solides, \\ Facultés Universitaires Notre-Dame de la Paix, 61 rue de Bruxelles, B-5000, Namur Belgium \\ J. Ghijsen, R. L. Johnson, and L. Ley \\ Max-Planck-Institut für Festkörperforschung, Heisenbergstrasse 1, \\ Postfach 8006 65, D-7000 Stuttgart 80, Federal Republic of Germany \\ (Received 26 November 1986)
}

\begin{abstract}
We have studied the interface formation between $\mathrm{Sb}$ (111) surfaces and evaporated aluminum with photoemission using synchrotron radiation. Energy distribution curves were measured from the Al $2 p$ and $\mathrm{Sb} 4 d$ core levels and from the valence band. A curve-fitting procedure was applied to the core-level spectra in order to decompose the peaks into individual components. A model calculation was performed to explain the intensity variation of the different $\mathrm{Al} 2 p$ components. We show that a two-dimensional layer of $\mathrm{AlSb}$ is formed and that $\mathrm{Al}$ clusters grow on top of $\mathrm{AlSb}$ as soon as a fraction of a monolayer of $\mathrm{AlSb}$ is present. This also explains the behavior of the $\mathrm{Sb} 4 d$ integrated peak areas and of the valence-band energy distribution curves. Finally, partial-yield spectra have been measured in order to assess the AlSb formation and to study the $\mathrm{Al} 2 p$ exciton.
\end{abstract}

\section{INTRODUCTION}

The study of solid-state interfaces is a topic of increasing interest. Modern microelectronic circuits include a wide variety of materials whose interfaces are of crucial importance in determining the device characteristics. ${ }^{1,2}$ Such devices are often built following empirical recipes, and sometimes only little is known about the exact interface structure and composition. Nowadays, the availability of modern evaporation techniques combined with interface sensitive spectroscopies such as photoemission employing synchrotron radiation allows a detailed investigation of the formation of these interfaces and provides an identification and location of the chemical species that are formed. Until now, much effort has been concentrated on the study of metal-semiconductor contacts, ${ }^{1,2}$ especially for those interfaces involving silicon or compound semiconductors such as GaAs (Refs. 3 and 4) and InP (Refs. 5 and 6) that are widely used in microelectronic applications. III-V compound semiconductors with antimony as the group- $\mathrm{V}$ element are also interesting for certain technological applications as well as for more fundamental studies of quantum well structures. ${ }^{7,8}$

In this paper, we study the formation of a III-V compound semiconductor interface between a group-III metal element (aluminum) and a group-V semimetal (antimony). The study is based on the analysis of the $\mathrm{Al} 2 p$ and $\mathrm{Sb} 4 d$ core-level spectra as well as of the valence-band energy distribution curves (EDC's) recorded during the deposition of $\mathrm{Al}$ on $\mathrm{Sb}(111)$ single-crystal surfaces. We will show that a disordered monolayer of AlSb is formed, covered with metallic Al clusters. These clusters start to develop as soon as a fraction of a monolayer of $\mathrm{AlSb}$ is present.

In an earlier work Delrue et al. ${ }^{9}$ have studied the growth of indium on $\mathrm{Sb}(111)$ single crystals at room tem- perature with Auger electron spectroscopy, low-energy electron diffraction (LEED) and x-ray photoelectron spectroscopy. A Stranski-Krastanov-type growth was observed in that case with InSb being formed in the first monolayer.

The work of Noreika et al. ${ }^{10}$ who studied antimony on $\operatorname{InSb}(111)$ is also worth mentioning in this context. They found it possible to grow smooth epitaxial films of $\mathrm{Sb}$ on $\mathrm{InSb}(111)$ surfaces at $550 \mathrm{~K}$ despite the lattice mismatch of $6 \%$. By comparison, the lattice mismatch of $\mathrm{AlSb}$ on $\mathrm{Sb}(111)$ is only $0.3 \%$, and epitaxy might thus be expected. We did not, however, observe such an epitaxial growth in the present room-temperature study.

\section{EXPERIMENTAL TECHNIQUES}

The experimental setup used for this study was a photoelectron spectrometer at the FLIPPER-2 monochromator on the DORIS (Doppel-Ring Speicheranlage) storage ring at the synchrotron radiation laboratory HASYLAB (Hamburger Synchrotronstrahlungslabor, Deutsches Elektronensynchrotron DESY, Hamburg). A detailed description can be found elsewhere. ${ }^{11}$ The UHV system has three parts: a small introduction chamber, a large preparation chamber, and an analysis chamber with base pressures in the range of $10^{-8}-10^{-9} \mathrm{~Pa}$, respectively.

$\mathrm{Sb}$ (111) surfaces were prepared in the analysis chamber by cleaving oriented crystals from Metal Crystals and Oxides Ltd. (Cambridge, England) and from the MaxPlanck-Institut (Stuttgart, Germany). The cleanliness and the crystallographic quality of these surfaces were checked with soft-x-ray photoemission (SXPS) and LEED. Aluminum deposition was carried out in the sample preparation chamber from a molecular-beam epitaxy effusion cell at a low rate $(\cong 1 \AA / \mathrm{min})$ and at a pressure of $5 \times 10^{-8} \mathrm{~Pa}$. Careful outgassing of the cell prior to the 
experiment and liquid-nitrogen cooling of a cryopanel during the Al deposition was necessary to maintain such a low pressure. The evaporation rate was calibrated with a quartz thickness monitor which could be brought to the same position as the sample. The Al coverage $\theta$ will be expressed in monolayer units (ML) throughout this work. One monolayer is defined as the density corresponding to the surface density of $\mathrm{Sb}$ atoms on the $\mathrm{Sb}(111)$ surface: 1 $\mathrm{ML}=2.16 \times 10^{15} \mathrm{Sb}$-atoms $\mathrm{cm}^{-2}=3.6 \AA$.

\section{EXPERIMENTAL RESULTS}

Three different batches of samples were tried before satisfactory mirrorlike cleaved surfaces were obtained. The clean surface exhibits a sharp hexagonal LEED pattern. The valence-band energy distribution curve (EDC) recorded for $h v=44 \mathrm{eV}$ shown in Fig. 1 (lower curve) is representative of the cleaved surface.

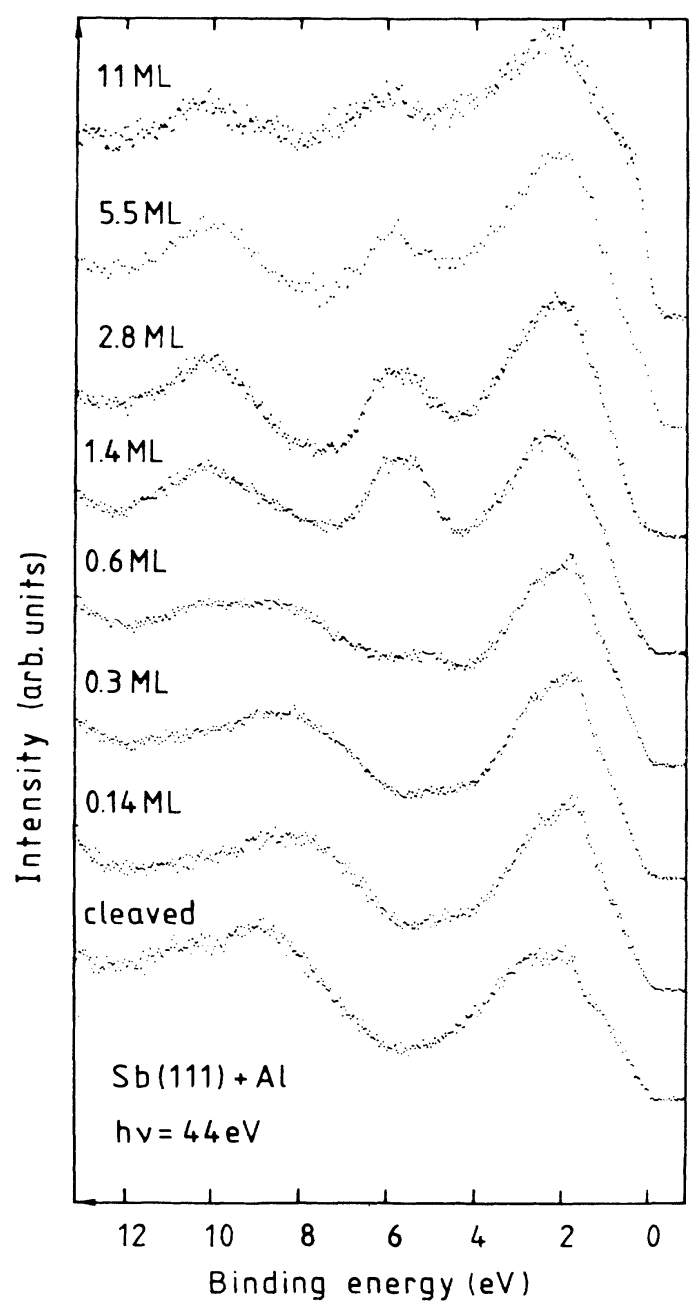

FIG. 1. Valence-band EDC's from $\mathrm{Sb}(111)$ with increasing aluminum coverage.

\section{A. Valence-band spectra}

Energy distribution curves from the valence band have been measured from the cleaved surface and for different $\mathrm{Al}$ coverages at a photon energy of $44 \mathrm{eV}$ yielding maximum surface sensitivity. The results are shown in Fig. 1. In the low coverage region ( $0-1$ monolayer), only minor changes are seen. In particular, the density of states close to the Fermi level remains low, which shows that the aluminum is in a nonmetallic phase at low coverages. An additional structure appears at (5.5-6.0)-eV binding energy, which coincides with a maximum in the valence density of states in AlSb. ${ }^{12}$ At 1.4 ML coverage, the valenceband EDC is very similar to the one observed by Johnson et al. on polycrystalline AlSb. ${ }^{13}$ At higher Al coverages $(\theta \geq 3 \mathrm{ML})$, a metallic Fermi edge develops. However, even at coverages as high as 11 monolayers, the structure of the AlSb valence band is still visible. As the valence band from pure Al has no pronounced features except for a peak close to the Fermi edge, ${ }^{14}$ we suggest that aluminum forms clusters at these coverages, leaving parts of the underlying AlSb layer uncovered. This possibility will be examined in detail in a subsequent section.

It might be argued that the $5.5-6.0 \mathrm{eV}$ valence-band feature is close to where an oxide feature is expected. In fact, we have identified such oxygen-induced peaks from contaminated Sb samples covered or not with aluminum. In such cases, the oxide feature was always observed at (6.5-7.5)-eV binding energy and its width is about two times larger than the width of the AlSb peak.

\section{B. Core-level spectra}

\section{Al $2 p$ core level EDC's}

Energy distribution curves from the Al $2 p$ core level have been measured with a photon energy of $100 \mathrm{eV}$ selected for maximum surface sensitivity $(\lambda \cong 5-10 \AA) .{ }^{15}$ This excitation energy also yields maximum intensity for the Al $2 p$ core line. ${ }^{16}$ Figure 2 shows the sequence of $\mathrm{Al}$ $2 p$ EDC's obtained at different aluminum coverages ranging from 0 to 22 monolayers. The data have been smoothed, the background has been subtracted, and the curves have been plotted to equal height. The bindingenergy scale is referred to the Fermi level $\left(E_{F}\right)$ which was measured directly for high Al coverages. At low coverages the Fermi level coincides with the top of the valence band of the Sb substrate. ${ }^{17,18}$ The Al $2 p$ binding energy for bulk metallic aluminum [72.71 $\pm 0.05 \mathrm{eV}$ (Ref. 19)], as well as for polycrystalline AlSb $(73.04 \pm 0.10 \mathrm{eV})$, are marked with arrows in Fig. 2. A detailed analysis of these results will be given below after fitting individual spinorbit pairs to the data. However, certain general trends can already be observed. At low coverages a broad peak is seen at higher binding energies as compared to metallic aluminum. This peak is relatively symmetric and no spin-orbit splitting is resolved, so one must assume that at least two components are present. The mean binding energy of the Al $2 p$ level gradually increases up to $2.8 \mathrm{ML}$ coverage, whereas at higher coverages metallic aluminum 
becomes clearly visible. The binding-energy difference between the metallic Al component and the mean position of the Al $2 p$ peak at low coverages is of the order of $0.5-1 \mathrm{eV}$, which is a typical value for III-V semiconductor formation with $\mathrm{Al}^{20}$

\section{2. $S b 4 d$ core-level EDC's}

Energy distribution curves from the $\mathrm{Sb} 4 d$ core level have been measured with a photon energy of $70 \mathrm{eV}$ so that the escape depth is similar to that of $\mathrm{Al} 2 p$. The sequence of spectra obtained at aluminum coverages from 0 to 22 monolayers is shown in Fig. 3. It is obvious from these spectra that an additional peak starts to develop at the low-binding-energy side even at the lowest coverages used in this experiment. This new component, which becomes more important with increasing Al coverage, is attributed to AlSb formed at the surface.

More bulk-sensitive spectra have been recorded with a

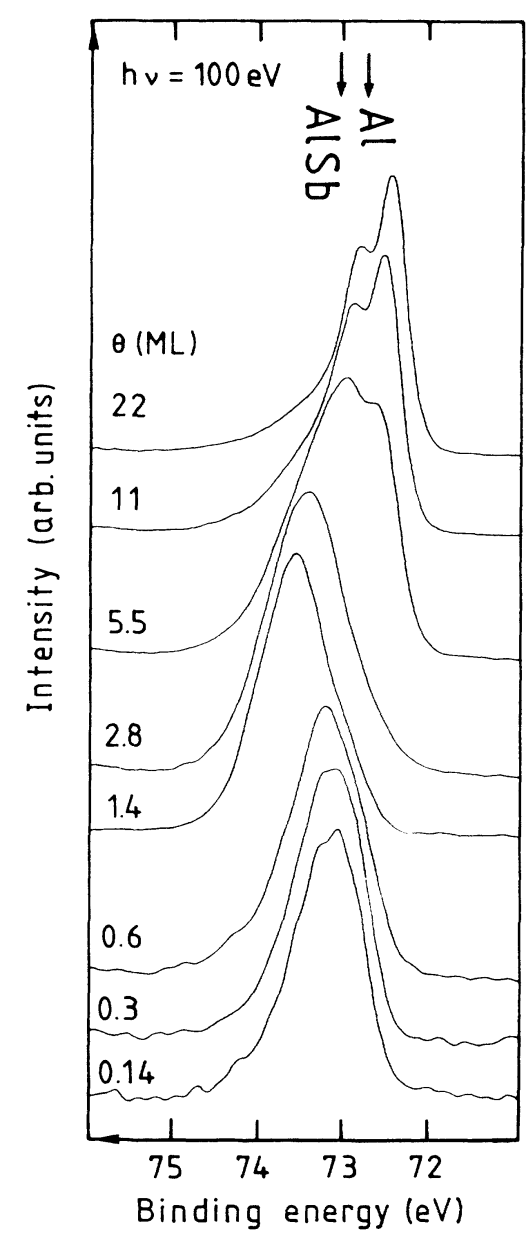

FIG. 2. Al $2 p$ core-level energy distribution curves from $\mathrm{Sb}(111)$ at different $\mathrm{Al}$ coverages. The binding-energy scale refers to the Fermi level. After subtraction of a polynomial background, the spectra have been normalized to equal height. The arrows indicate the binding energies for metallic Al and AlSb. photon energy of $42 \mathrm{eV}$. Figure 4 shows a comparison between some EDC's from clean $\mathrm{Sb}$ (111) surfaces (solid line) and from the same sample covered with 1.4 ML of aluminum (dotted line), measured with a photon energy of 70 $\mathrm{eV}$ and $42 \mathrm{eV}$, respectively.

Although the $I\left(4 d_{5 / 2}\right) / I\left(4 d_{3 / 2}\right)$ intensity ratio is close to the statistical value of $3: 2$ in the $70-\mathrm{eV}$ spectra, it strongly deviates from that value in the case of $h v=42$ $\mathrm{eV}$. Since this value is close to the Sb $4 d$ edge, the photoionization cross section increases very rapidly with increasing photon energy, and this affects the branching ratio. ${ }^{21}$ Another difference between the surface-sensitive $70-\mathrm{eV}$ spectra and the more bulk-sensitive $42-\mathrm{eV}$ spectra is the fact that the additional component previously identified as AlSb is strongly reduced in the $42-\mathrm{eV}$ spectra, indicating that mainly AlSb is present at the surface.

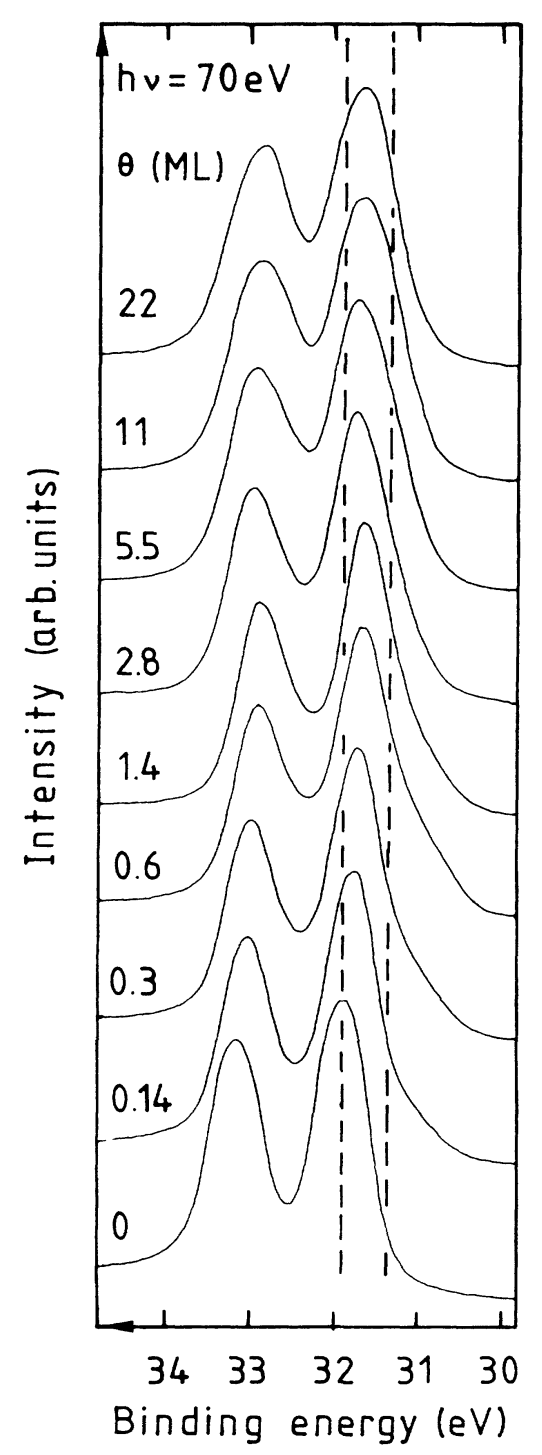

FIG. 3. Sb $4 d$ core-level EDC's from $\mathrm{Sb}(111)$ with increasing Al coverage. The binding-energy scale refers to the Fermi level. The spectra have been normalized to equal height. 


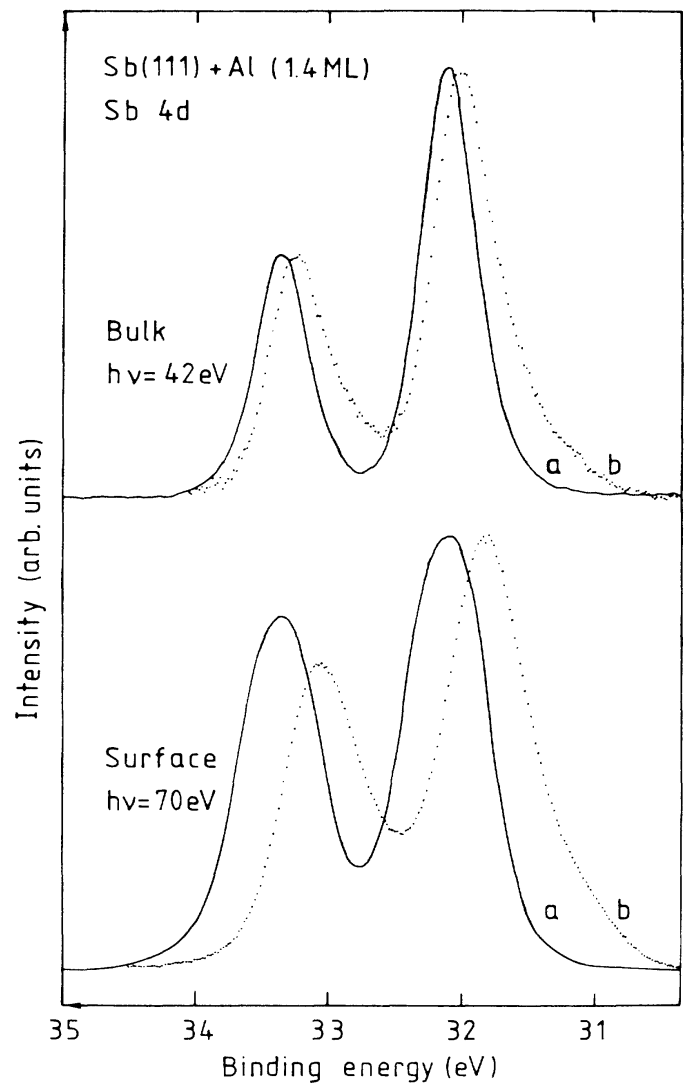

FIG. 4. Sb $4 d$ core-level EDC's from clean $\mathrm{Sb}(111)$ surfaces (solid line) and from $\mathrm{Sb}(111)+1.4 \mathrm{ML} \mathrm{Al}$ (dotted line) measured at different photon energies.

\section{DISCUSSION}

In order to obtain more information from the core-level spectra, we have analyzed the $\mathrm{Al} 2 p$ and Sb $4 d$ EDC's by fitting them to the smallest possible number of spin-orbit doublets (see Appendix). Some parameters were allowed to vary in rather narrow limits, due to physical reasons (e.g., the spin-orbit parameters of $\mathrm{Al}$ and $\mathrm{Sb}$ ). The photoemission lines were represented by either Doniach-Sunjić ${ }^{22}$ or Lorentzian curves convolved with a Gaussian, accounting for spectrometer resolution and other possible sources of inhomogeneous broadening. ${ }^{23}$ We used a unique Gaussian broadening for the different components in one spectral range (Al $2 p$ or $\mathrm{Sb} 4 d$ ) which is a reasonable approximation since we are interested in line intensities and positions. Typical fit results are shown in Fig. 5. The relevant fitting parameters are summarized in Table $I$ and in Figs. 6-11. The validity of our approach is confirmed by the systematic behavior of our results and by the convergence of the Al $2 p$ binding energy to that of bulk Al at high coverages.

\section{A. Sb $4 d$ and Al $2 p$ core level}

For the $\mathrm{Sb} 4 d$ core-level spectra, individual spin-orbit doublets have been obtained by the convolution of
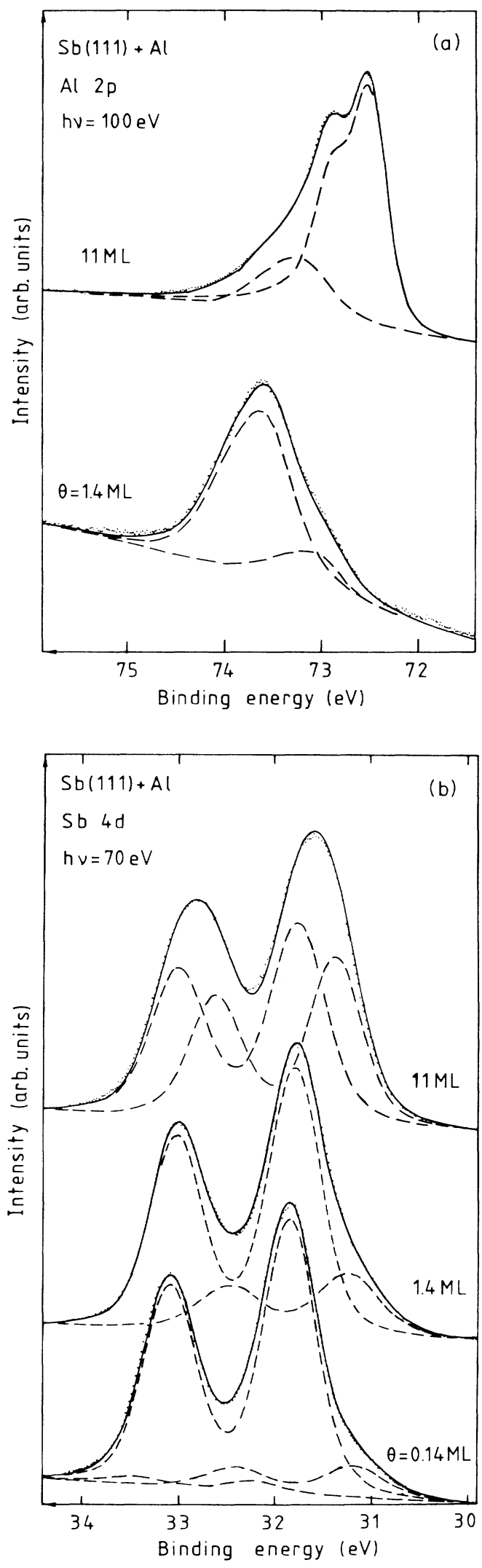

FIG. 5. Typical results from curve fitting to the Al $2 p$ corelevel EDC's (a), and the Sb $4 d$ core-level EDC's (b). 
TABLE I. Parameters used for the fits to the core level spectra explained in the text. We have used a Gaussian broadening of $0.315 \pm 0.015 \mathrm{eV}$ for the $\mathrm{Al} 2 p$ spectra and of $0.500 \pm 0.020 \mathrm{eV}$ for the $\mathrm{Sb} 4 d$ spectra. Branching ratio was 0.50 for all the $\mathrm{Al} 2 p$ spectra and $0.725 \pm 0.005$ for $\mathrm{Sb} 4 d$. The binding energies as well as the intensities of the peaks are shown in Figs. $6-10$.

\begin{tabular}{cccccc}
$\begin{array}{c}\text { Coverage } \\
(\mathrm{ML})\end{array}$ & $\begin{array}{c}\text { Al-1 } \\
\text { FWHM } \\
(\mathrm{meV})\end{array}$ & $\alpha$ & $\begin{array}{c}\text { Al-2 } \\
\text { FWHM } \\
(\mathrm{meV})\end{array}$ & $\begin{array}{c}\text { Sb-1 } \\
\text { FWHM } \\
(\mathrm{meV})\end{array}$ & $\begin{array}{c}\text { Sb-2 } \\
\text { FWHM } \\
(\mathrm{meV})\end{array}$ \\
\hline 0.14 & 190 & 0.123 & 290 & 93 & \\
0.28 & 150 & 0.122 & 310 & 135 & 170 \\
0.56 & 150 & 0.122 & 300 & 130 & 180 \\
1.39 & 210 & 0.123 & 270 & 125 & 200 \\
2.78 & 210 & 0.125 & 270 & 110 & 210 \\
5.56 & 100 & 0.125 & 290 & 130 & 150 \\
11.1 & 70 & 0.122 & 290 & 110 & 190 \\
22.2 & 44 & 0.123 & 200 & 120 & 150 \\
\hline \hline
\end{tabular}

Lorentzian profiles with Gaussians. Doniach-Šnjić line shapes are not required due to the relatively low density of states close to the Fermi level. The Gaussian broadening was of $0.5 \mathrm{eV}$ for the $\mathrm{Sb} 4 d$ lines, which is larger than the overall spectrometer resolution $(\cong 0.33 \mathrm{eV})$. For clean $\mathrm{Sb}$ (111) surfaces, two unresolved components are already needed to describe the data. The first $\mathrm{Sb} 4 d_{5 / 2}$ component is found at $32.05 \pm 0.10 \mathrm{eV}$ below the Fermi level and will be called Sb-1 in the following. We identify the Sb-1 component with bulk $\mathrm{Sb}$. The second component, about eight times weaker, observed at $32.30 \pm 0.10 \mathrm{eV}$ below the Fermi level, is probably due to a surface shift. ${ }^{19,24}$ In the present case, the surface shift is most probably due to different screening of the core hole in the bulk and at the surface, which increases the measured binding energy at the surface. This means that the introduction of a second component is just a way of accounting for several unresolved peaks in the present case. ${ }^{25}$

As soon as a fraction of a monolayer of aluminum is deposited on the $\mathrm{Sb}$ (111) surface, a new component (Sb-2) must be included at $570 \pm 50 \mathrm{meV}$ lower binding energy compared to the $\mathrm{Sb} 4 d$ bulk value. This separation decreases with increasing Al coverage. The spin-orbit parameters used for the fits were $\Delta_{\text {s.o. }}=1.24 \mathrm{eV}$ and $I\left(4 d_{3 / 2}\right) / I\left(4 d_{5 / 2}\right)=0.72 \pm 0.02$ for all the components at any coverage. The intensity ratio is relatively close to the statistical ratio of $2: 3$, the slight difference being probably due to photoionization cross-section effects. ${ }^{21}$

To fit the Al $2 p$ core-level spectra, we needed one Lorentzian component folded with a Gaussian of $315 \pm 15$ meV full width at half maximum (FWHM) and one Doniach-Sunjic line folded with the same Gaussian. At high Al coverage this latter component is found at $72.50 \pm 0.10 \mathrm{eV}\left(\mathrm{Al} 2 p_{3 / 2}\right)$ below the Fermi level, which is close to the binding energy of metallic aluminum. ${ }^{19}$ The asymmetry parameter for this component was $\alpha=0.122 \pm 0.03$ at any coverage and the lifetime width decreased monotonically from 190 to $44 \mathrm{meV}$. A comparison of these values with results from Citrin et al. ${ }^{26}$ shows that this component is characteristic of bulk aluminum at high coverages. The other component, whose lifetime width is much larger (between 200 and $300 \mathrm{MeV}$ dependent on the coverage), is shifted by $0.6 \pm 0.1 \mathrm{eV}$ towards higher binding energies. These components will be referred to as Al-1 (lower binding energy) and Al-2 (higher binding energy), respectively.

We have shown in Fig. 6 the intensity variation of the three $\mathrm{Sb} 4 d$ components as well as the total intensity (solid dots) versus Al coverage. Figure 7 shows the corresponding results for the Al $2 p$ spectra. Upon Al coverage $\mathrm{Sb}-2$ grows rapidly in intensity, reaches a maximum around $3 \mathrm{ML}$, and drops off beyond as more aluminum is added. This behavior is paralleled by the Al-2 component, and we ascribe these two components therefore to AlSb that is formed upon the initial coverage of the $\mathrm{Sb}(111)$ surface. This also explains the small total $\mathrm{Sb}$ intensity variations up to $3-\mathrm{ML}$ coverage. We also remember that AlSb compound formation is obvious from the valence-band spectra, especially at 1-2 monolayer coverage. At intermediate coverages, the "bulk" Sb signal decreases, whereas the chemically shifted component is of almost constant intensity. We therefore suggest that the amount of AlSb increases, but AlSb simultaneously covers up with unreacted aluminum, resulting in a decrease of the total $\mathrm{Sb} 4 d$ intensity. The almost linear decrease of all $\mathrm{Sb}$ components at $\theta>3 \mathrm{ML}$ and the corresponding increase of the Al-1 component can be explained by the growth of unreacted Al clusters on top of a complete AlSb layer covering the $\mathrm{Sb}(111)$ surface.

In summary, the behavior of the core-line intensities is explained by dividing the interface formation into three major regions: at low coverages (0-2 ML), AlSb formation is the predominant mechanism, at intermediate coverages ( $2-4 \mathrm{ML})$, we observe a competition between AlSb formation and the growth of metallic clusters, and at high coverages $(>4 \mathrm{ML})$ metallic Al-cluster growth is the predominant feature.

We shall close this section by comparing the behavior of these two Al components with the following model: Al-2 is characteristic of a reacted AlSb phase in a twodimensional monolayer. An impinging $\mathrm{Al}$ atom has a certain probability $P(\theta)$ for reacting and forming AlSb. Assuming a sticking coefficient of 1 , the same atom has a probability $1-P(\theta)$ for going into an aluminum cluster 


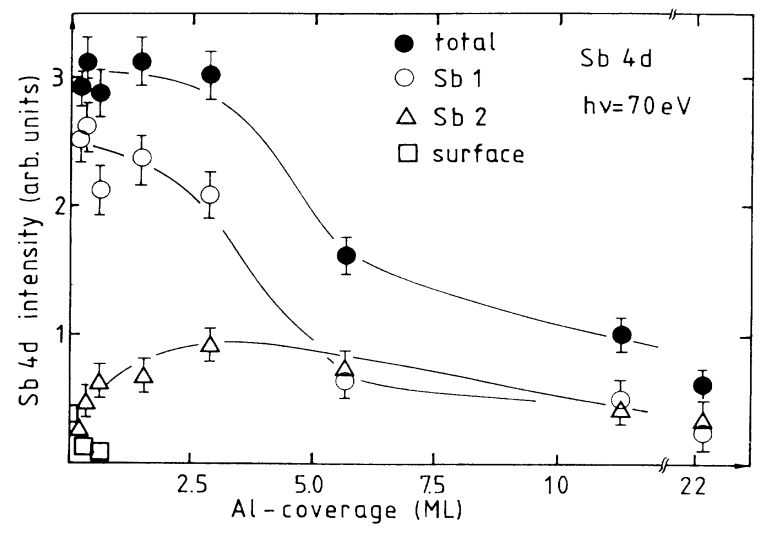

FIG. 6. Integrated peak areas from different components in the $\mathrm{Sb} 4 d$ spectra determined after curve fitting. Open circles: "bulk" Sb; open triangles: "AlSb"; open squares: Sb $4 d$ surface peak; and solid circles: sum of the three previous components. For details see text.

on top of the AlSb compound. If we assume that the probability for an impinging atom to form AlSb is proportional to the area of the unreacted surface, a straightforward calculation yields for this probability the simple form

$$
P(\theta)=\exp (-\theta) .
$$

The probability $P(\theta)$ decreases as the surface covers up with AlSb. Due to this decrease of $P(\theta)$, the surface covers up slower when $\theta$ increases, which slows down the decrease of $P(\theta)$. Assuming that the average cluster concentration $N_{c}$ is constant as long as the total area covered by the clusters is small compared to the sample surface, and assuming a hemispherical shape for the clusters, we can follow a procedure similar to the one used by Franciosi et al. ${ }^{27}$ to derive the intensities for the Al $2 p$ core-

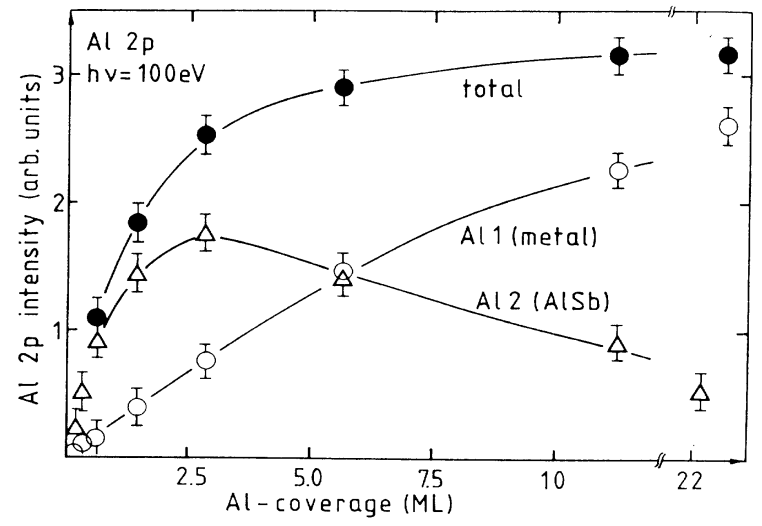

FIG. 7. Integrated peak area from different components in the $\mathrm{Al} 2 p$ spectra determined after curve fitting. Open circles: Al-1 component; open triangles: Al-2 component. Solid circles: sum of both components. For details see text. level emission from the different components. The cluster radius $R$ is related to the coverage $\theta$ through the relation

$$
R(\theta)=\left(\frac{3 d[\theta-G(\theta)]}{2 \pi N_{c}}\right)^{1 / 3},
$$

where $d$ is the thickness corresponding to $1-\mathrm{ML}$ coverage and

$$
G(\theta)=\int_{0}^{\theta} P\left(\theta^{\prime}\right) d \theta^{\prime}
$$

We obtain for the signal intensity from the AlSb compound

$$
I_{c}(\theta)=I_{c}^{0}(1) \frac{G(\theta)}{G(1)}\left(1-N_{c} \pi R^{2}+2 N_{c} \pi \alpha\right)
$$

with

$$
\alpha=\lambda_{e}^{2}-\exp \left(-R / \lambda_{e}\right)\left(R \lambda_{e}+\lambda_{e}^{2}\right) .
$$

The effective escape depth $\lambda_{e}$ is related to the escape depth $\lambda$ by the relation

$$
\lambda_{e}=\lambda \cos \beta
$$

with $\beta$ being the mean acceptance angle of the analyzer $(\cos \beta \cong 0.74)$. For the contribution from the clusters, we obtain

$$
I_{m}(\theta)=k \pi N_{c} \lambda_{e}\left(R^{2}-2 \alpha\right)
$$

$k$ being a parameter. We have used these relations with parameters $k, \lambda_{e}, I_{c}^{0}(1)$, and $N_{c}$ in order to model the behavior of the different Al $2 p$ components. The result is shown in Fig. 8. The parameters have been determined by a nonlinear least squares fit to our data. The electron escape depth obtained from this fit is $\lambda \cong 6 \AA$. This value is obtained by neglecting refraction and diffraction effects. ${ }^{24}$ We find an average cluster density of $N_{c}=2.4 \times 10^{11}$ $\mathrm{cm}^{-2}$.

It is important to realize that this fit has been obtained with the same values for the parametes for both the signal from the two-dimensional monolayer and the metallic cluster contribution. We also emphasize that this model is in agreement with the general behavior of the $\mathrm{Sb} 4 d$ core-level spectra as described in an earlier section, as well as with our observation from the valence bands.

\section{B. Core-level binding energies}

The Al $2 p$ core-level binding energies were measured with respect to the Fermi level. They are shown versus Al 


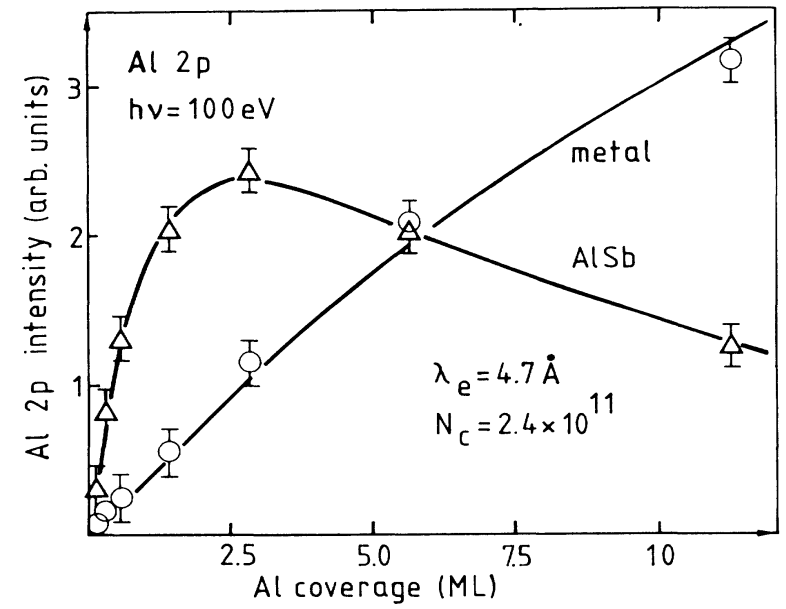

FIG. 8. Comparison between the experimental results from Fig. 7 and the theoretical model explained in this work. Circles: Al-1 component. Triangles: Al-2 component.

coverage in Fig. 9. For this discussion, we shall consider the Al atoms to form clusters of $n^{\prime}$ atoms, with $m=n^{\prime}-n$ atoms in contact with the substrate. The binding-energy changes of components Al-1 and Al-2 are then expected to be roughly the same with Al-2 being shifted with respect to Al-1 due to different chemical environment.

We will focus our attention now on the behavior of the lower-binding-energy component Al-1. At the beginning of the interface formation, this Al-1 component is found at a binding energy intermediate between the values for bulk metallic $\mathrm{Al}$ and $\mathrm{Al}$ bonded to the Sb substrate. The binding energy then increases by $200 \mathrm{meV}$ up to a maximum at 1.4-ML coverage, decreasing again and converging to the value for metallic Al at sufficiently high coverages. We will show now that the initial binding-energy increase can be explained in terms of the gradual transition

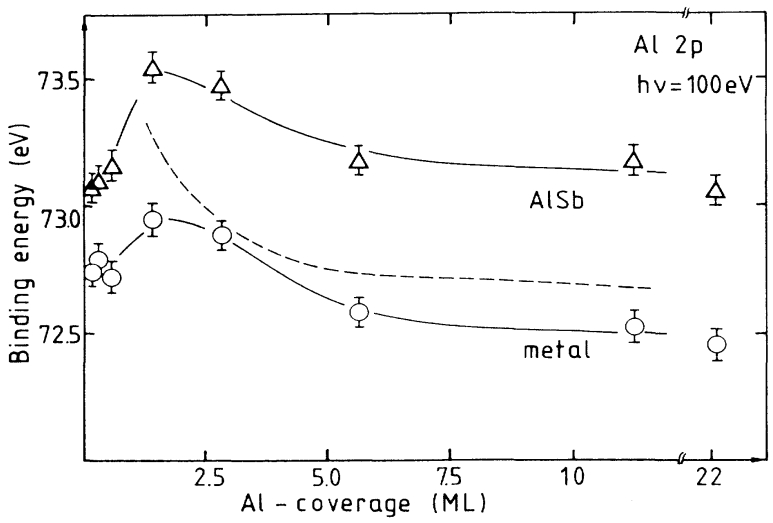

FIG. 9. Binding energies of the two peaks in the Al $2 p$ spectra determined after curve fitting. Open circles: Al-1 component; Open triangles: Al-2 component. For details see text. from small $\mathrm{Al}_{n}$ molecular clusters to metallic clusters. The final decrease will be intepreted by final-state effects.

A detailed theoretical study of small Al clusters on GaAs(110) has been performed by Zunger. ${ }^{28}$ He showed that the clusters are generally nonmetallic until a critical size of about 50-150 atoms is reached, corresponding to a radius of typically $10 \AA$ in the case of hemispherical clusters. In the nonmetallic case, his model calculation predicts a binding energy for the Al $2 p$ core level between the bulk metallic value and the value corresponding to bonding to the substrate, which is exactly what we observe at the lowest coverages. In the case of metallic clusters supported on insulating substrates, Wertheim et al. ${ }^{29,30}$ have shown that positive binding-energy shifts with decreasing cluster size can be explained by the Coulomb energy $e^{2} / r$ appearing on the cluster due to screening of the core hole by conduction electrons. On a semimetal substrate this charge is not neutralized during the time relevant for photoemission, but the shift is lowered due to screening via the formation of an image charge in the substrate, especially when the cluster becomes larger. ${ }^{30}$ The shift thus decreases faster than the $1 / r$ behavior in free clusters. The dashed line in Fig. 8 represents this $e^{2} / r$ behavior. We conclude that our measured values reflect the predicted trends. At coverages lower than $3 \mathrm{ML}$ the shift saturates in agreement with earlier predictions. ${ }^{29,30}$ At coverages below $0.3 \mathrm{ML}$ the cluster radius deduced from relation (2) is smaller than $15 \AA$, and the cluster can thus be considered to be nonmetallic. The initial bindingenergy increase is then understood as a gradual transition from nonmetallic to metallic clusters.

In addition to the binding-energy shift, the Al $2 p$ corelevel spectra show an increased width at low cluster size, as already reported in the case of Sn (Ref. 30) and Ag clusters (Ref. 31). This width change reflects the size distribution of clusters during the Al deposition.

The binding energy of the $\mathrm{Sb} 4 d$ core level with respect to the Fermi level is shown versus coverage in Fig. 10. The larger error bars for the "surface" peak are due to its

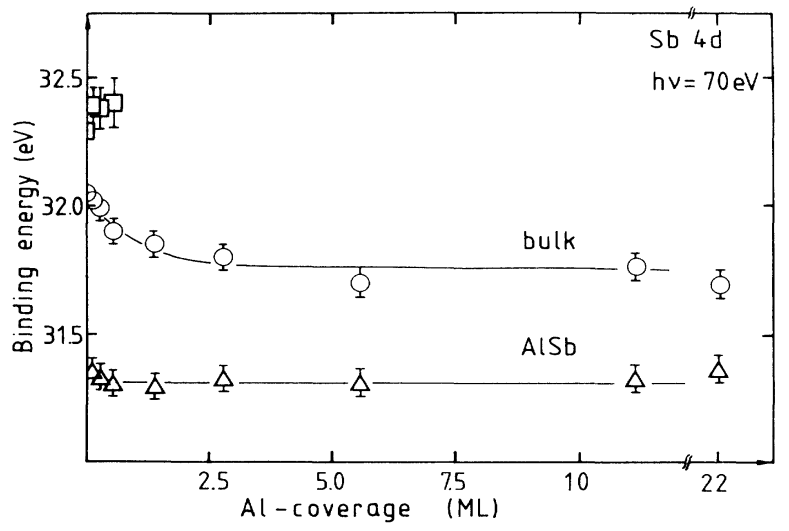

FIG. 10. Binding energies of the different components in the $\mathrm{Sb} 4 d$ spectra determined after curve fitting. Open circles: "bulk" Sb; open triangles: "AlSb"; open squares: Sb $4 d$ surface peak. For details see text. 
comparatively low intensity and to the fact that this peak is not resolved from the bulk peak. As an explanation of the binding-energy decrease for the unreacted component we suggest that even $\mathrm{Sb}$ atoms from subsurface layers are polarized due to the AlSb formation at the surface.

\section{Partial-yield spectra}

The aluminum antimonide formation of $\mathrm{Sb}(111)$ single crystals is confirmed by partial-yield spectroscopy. Figure 11 shows the partial-yield spectrum measured on $\mathrm{Sb}$ (111) covered with 0.1 monolayers of $\mathrm{Al}$ at a final energy $E^{*}=60 \mathrm{eV}$ at the Al $2 p$ absorption edge. A sharp doublet is observed with a linewidth comparable to the overall spectrometer resolution. We have analyzed similar spectra after annealing a $100-\AA$ thick $\mathrm{Al}$ overlayer on $\mathrm{Sb}(111)$ at $600 \mathrm{~K}$. The final energy was equal to $6 \mathrm{eV}$ in this latter case. The separation between both peaks is 0.39 $\mathrm{eV}$ and the maximum of the first peak lies at $74.40 \mathrm{eV}$. The intensity ratio of the two components is very close to 1. A doublet with the same characteristics has also been observed by Johnson et al. on polycrystalline AlSb (Ref. 13) and was attributed to bulk excitons associated with the lowest conduction band. The Al $2 p$ binding energy of the AlSb component with respect to the top of valence band can be used to estimate the difference between the gap and the exciton binding energy. We obtain $E_{g}-E_{\text {ex }}=1.19$ $\pm 0.15 \mathrm{eV}$, which agrees with the result of Johnson et al. $(1.45 \mathrm{eV})$ within the experimental errors.

\section{CONCLUSIONS}

We have studied the room-temperature growth of aluminum on $\mathrm{Sb}(111)$ single crystals with photoemission using synchrotron radiation. We have shown that the interface formation can be described by a rather simple

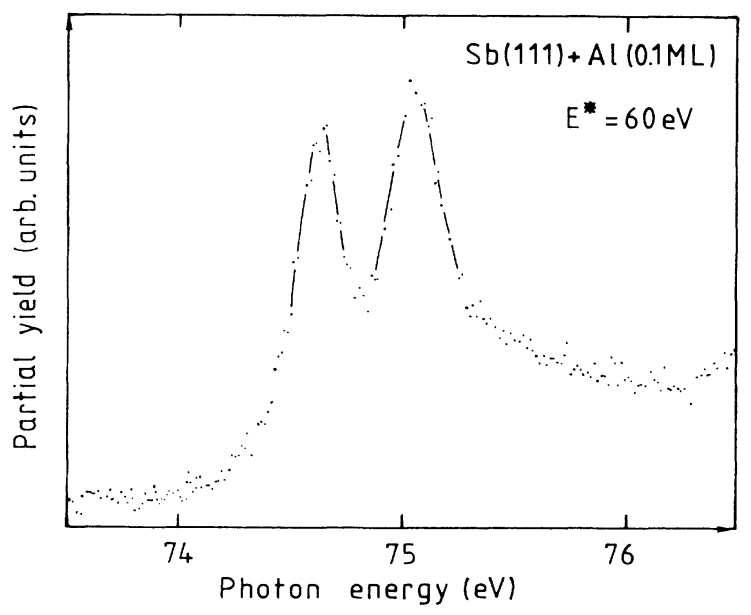

FIG. 11. Partial-yield spectrum from $\mathrm{Sb}(111)+0.1$ monolayers $\mathrm{Al}$ at the $\mathrm{Al} 2 p$ absorption edge. model. An impinging atom has a probability $P(\theta)$ to react and form AlSb. Otherwise, it goes into Al clusters on top of the AlSb layer. This means that the AlSb formation is the predominant mechanism at low coverages, whereas at high coverages $\mathrm{Al}$ cluster formation is more pronounced. The $\mathrm{Al} 2 p$ core-level intensity variations are in quantitative agreement with this model, which also qualitatively explains the behavior of the $\mathrm{Sb} 4 d$ core levels and the valence band EDC's. The Al $2 p$ binding-energy variation can be explained in two steps: An initial increase is due to the transition from very small nonmetallic clusters to metallic clusters, whereas the following decrease is explained by the Coulomb energy $e^{2} / r$ appearing on the cluster due to screening of the core hole by conduction electrons. The AlSb formation has been further confirmed by partial-yield spectroscopy. On clean $\mathrm{Sb}(111)$ samples a surface shift is detected from the $\mathrm{Sb} 4 d$ corelevel EDC's and is attributed to different screening in the final state in the bulk and at the surface.

Further investigations on the influence of the temperature on the interfacial behavior and measurements of the core-level angular distributions are currently in progress to complete our understanding of the interaction mechanism.

\section{ACKNOWLEDGMENTS}

We are grateful to M. Cardona, A. A. Lucas, and J. P. Vigneron for comments and discussions as well as to $\mathbf{M}$. Taniguchi for his assitance during the measurements. This work is supported by the National Belgian Foundation for Scientific Reserach (FNRS) and by the German Bundesminister für Forschung und Technologie (BMFT) under Project No. $05390 \mathrm{CAB}$ and $05250 \mathrm{Ca}$. We acknowledge the National Belgian Foundation for Scientific Research (FNRS), IBM Belgium, and the Facultés Universitaires Notre-Dame de la Paix (FNDP) for the use of the Namur Scientific Computing Facility. One of us (E.P.) was supported in part by Institut de Recherches Supéricures Industrielles et Ayricotes (IRSIA); another (J.J.P.), by the Belgian FNRS.

\section{APPENDIX}

Here we show the fitting procedure for the SXPS corelevel EDC's. After subtraction of a linear background, the experimental spectra, $Y_{\text {mes }}(E)$, are fitted to the sum of individual components of the form

$$
Y(E)=f(E)^{*} g(E),
$$

where $f(E)$ will be described hereafter and $g(E)$ is a Gaussian with full-width-at-half-maximum $G$.

$f(E)$ is the function derived by Doniach and Sunjić: ${ }^{22}$

$$
f(E)=H \frac{\left.\cos (\pi \alpha / 2)+(1-\alpha) \arctan \left[\left(E-E_{0}\right) / \gamma\right)\right]}{\left[\left(E-E_{0}\right)^{2}+\gamma^{2}\right]^{(1-\alpha) / 2}} .
$$


When $\alpha=0$, a Lorentzian is obtained. This is generally the case if the density of states close to the Fermi level is low, i.e., in our case for $\mathrm{Sb}$ and $\mathrm{AlSb}$, whereas $\alpha$ is nonzero for metallic Al.

For each peak, the fitting parameters are thus $H, \alpha, \gamma$, and $E_{0}$, whereas a unique parameter $G$ was used for the different components in one spectral range (Al $2 p$ or $\mathrm{Sb}$ $4 d$ ). Additionally, for each doublet, the spin-orbit parameters (splitting and branching ratio) were allowed to vary in narrow limits. The fitting parameters were adjusted to minimize the chi-square

$$
\chi^{2}=\frac{1}{n-f} \sum_{i=1}^{n} \frac{\left[Y\left(E_{i}\right)-Y_{\mathrm{mes}}\left(E_{i}\right)\right]^{2}}{Y_{\mathrm{mes}}\left(E_{i}\right)}
$$

$n$ being the number of data points and $f$ being the number of fitting parameters.
${ }^{1}$ D. L. Lile, J. Vac. Technol. B 2, 496 (1984).

${ }^{2}$ D. K. Ferry, J. Vac. Sci. Technol. B 2, 504 (1984).

${ }^{3}$ A. Kahn, J. Carelli, D. Katnani, C. B. Duke, A. Paton, and L. J. Brillson, J. Vac. Sci. Technol. 19, 331 (1981).

${ }^{4}$ L. J. Brillson, R. S. Bauer, R. Z. Bachrach, and G. Hansson, Appl. Phys. Lett. 36, 326 (1984).

${ }^{5}$ A. Kahn, C. R. Bonapace, C. B. Duke, and A. Paton, J. Vac. Sci. Technol. B 1, 613 (1983).

${ }^{6}$ F. Houzay, P. Henoc, M. Bensoussan, and F. Barthe, J. Vac. Sci. Technol. B 3, 1212 (1985).

${ }^{7}$ H. Ploog, Y. Ohomoro, H. Okamoto, W. Stolz, and J. Wagner, Appl. Phys. Lett. 47, 384 (1985).

${ }^{8}$ R. G. van Welzenis and B. K. Ridley, Solid State Electron. 27, 113 (1984).

${ }^{9}$ J. P. Delrue, R. Sporken, and R. Caudano, J. Vac. Sci. Technol. A 4, 754 (1986).

${ }^{10}$ A. J. Noreika, M. H. Francombe, and C. E. C. Wood, J. Appl. Phys. 52, 7416 (1981).

${ }^{11}$ R. L. Johnson and J. Reichardt, Nucl. Instrum Methods 208, 791 (1983).

${ }^{12}$ N. J. Shevchik, J. Tejeda, and M. Cardona, Phys. Rev. B 9, 2627 (1974).

${ }^{13}$ R. L. Johnson, J. H. Fock, L. Ley, and M. Cardona, Proceedings of the 17th International Conference on the Physics of Semiconductors (Springer, Berlin, 1986), p. 1239.

${ }^{14}$ S. A. Flodström, L. G. Petersson, and S. B. M. Hagström, Solid State Commun. 19, 257 (1976).

${ }^{15}$ M. P. Seah and W. A. Dench, Surf. Interface Anal. 1, 2 (1979).

16J. J. Yeh and I. Lindau, At. Data Nucl. Data Tables 32, 2 (1985).

${ }^{17}$ L. M. Falicov and P. Lin, Phys. Rev, 141, 562 (1966).

18J. Robertson, Phys. Rev. B 28, 4671 (1983).
${ }^{19}$ R. Kammerer, J. Barth, F. Gerken, C. Kunz, S. A. Flodström, and L. I. Johansson, Phys. Rev. B 26, 3491 (1982).

${ }^{20}$ T. Kendelewicz, W. G. Petro, I. Lindau, and W. E. Spicer, Phys. Rev. B 30, 5800 (1984).

${ }^{21}$ G. Margaritondo, J. E. Rowe, and S. B. Christman, Phys. Rev. B 19, 2850 (1979); G. Margaritondo, R. Rosei, J. H. Weaver, and W. M. Becker, Solid State Commun. 34, 401 (1980).

${ }^{22}$ S. Doniach and M. Šunjić, J. Phys. C 3, 285 (1970).

${ }^{23}$ G. K. Wertheim, P. H. Citrin, and J. F. van der Veen, Phys. Rev. B 30, 4343 (1984).

${ }^{24}$ T. Miller, A. P. Shapiro, and T. C. Chiang, Phys. Rev. B 31, 7915 (1985).

${ }^{25} \mathrm{~A}$ simple relation can be obtained between the screening energy difference in the bulk and at the surface $E_{\mathrm{sc}}$ (surface bulk) and the dielectric constant $\epsilon_{\mathrm{Sb}}$ :

$$
E_{\mathrm{sc}}=\frac{1}{\epsilon_{\mathrm{Sb}}} \frac{\epsilon_{\mathrm{Sb}}-1}{\epsilon_{\mathrm{Sb}}+1} \frac{e^{2}}{2} .
$$

Using this relation we can estimate $\epsilon_{\mathrm{Sb}} \cong 21$.

${ }^{26}$ P. H. Citrin, G. K. Wertheim, and Y. Baer, Phys. Rev. Lett. 35, 885 (1975); Phys. Rev. B 16, 4256 (1977).

${ }^{27}$ A. Franciosi, D. J. Peterman, J. H. Weaver, and V. L. Moruzzi, Phys. Rev. B 25, 4981 (1982).

${ }^{28}$ A. Zunger, Phys. Rev. B 24, 4372 (1981).

${ }^{29}$ G. K. Wertheim, S. B. DiCenzo, and S. E. Youngquist, Phys. Rev. Lett. 51, 2310 (1983).

${ }^{30}$ G. K. Wertheim, S. B. DiCenzo, D. N. E. Buchanan, and P. A. Bennett, Solid State Commun. 53, 377 (1985).

${ }^{31}$ G. K. Wertheim, S. B. DiCenzo, and D. N. E. Buchanan, Phys. Rev. B 33, 5384 (1986). 